\title{
Genetic diversity and differentiation in Eryngium alpinum L. (Apiaceae): comparison of AFLP and microsatellite markers
}

\author{
M Gaudeul, I Till-Bottraud, F Barjon and S Manel \\ Laboratoire d'Ecologie Alpine, UMR CNRS 5553, Université J Fourier, BP 53, F-38041 Grenoble Cedex 09, France
}

\begin{abstract}
Genetic diversity and structure of 12 populations of Eryngium alpinum L. were investigated using 63 dominant amplified fragment length polymorphism (AFLP) and seven codominant microsatellite (48 alleles) markers. Within-population diversity estimates obtained with both markers were not correlated, but the microsatellite-based fixation index $F_{\text {is }}$ was correlated with both AFLP diversity indices (number of polymorphic bands and Nei's expected heterozygosity). Only AFLP diversity indices increased with the size of populations, although they did not significantly differ among them (Kruskall-Wallis test). The discrepancy between AFLPs and microsatellites may be explained by a better coverage of the genome with numerous AFLPs, the higher mutation rates of microsatellites or the absence of significant difference among withinpopulation diversity estimates. Genetic differentiation was higher with AFLPs $(\theta=0.40)$ than with microsatellites $(\theta=0.23)$, probably due to the higher polymorphism of
\end{abstract}

microsatellites. Thus, we considered global qualitative patterns rather than absolute estimates to compare the performance of both types of markers. On a large geographic scale, the Mantel test and multivariate analysis showed that genetic patterns were more congruent with the spatial arrangement of populations when inferred from microsatellites than from AFLPs, suggesting higher homoplasy of AFLP markers. On a small spatial scale, AFLPs managed to discriminate individuals from neighboring populations whereas microsatellites did not (multivariate analysis), and the percentage of individuals correctly assigned to their population of origin was higher with AFLPs than with microsatellites. However, dominant AFLPs cannot be used to study heterozygosity-related topics. Thus, distinct molecular markers should be used depending on the biological question and the geographical scale investigated.

Heredity (2004) 92, 508-518, advance online publication, 10 March 2004; doi:10.1038/sj.hdy.6800443

Keywords: microsatellites; AFLP; genetic diversity; genetic structure; assignment tests; conservation

\section{Introduction}

Biodiversity is composed of an inter- and an intraspecific component. Diversity assessments have long been performed by ecologists and were therefore often limited to species surveys, thus ignoring the intraspecific part of diversity (Till-Bottraud and Gaudeul, 2002). However, genetic variation between individuals and/or between populations is being increasingly investigated in domains such as forensics, management of genetic resources, crop development, evolutionary biology and conservation biology. Morphological traits and, later, allozymes and restriction fragment length polymorphism (RFLPs) were the first tools to evaluate diversity and differentiation. They are now widely replaced by new techniques that reveal diversity at the DNA level by using the polymerase chain reaction (PCR).

Among DNA-based markers, amplified fragment length polymorphism (AFLPs) and microsatellites are among the most informative. Although often used in the same scientific context (eg for the assessment of within-

Correspondence: M Gaudeul, Department of Plant Ecology, Evolutionary Biology Center (EBC), Uppsala University, Villavägen 14, Uppsala 752 36, Sweden.E-mail:myriam.gaudeul@ebc.uu.se

Received 21 June 2002; accepted 12 December 2003; published online 10 March 2004 and among-population genetic diversity), AFLPs and microsatellites present major distinctions. The AFLP technique (Vos et al, 1995) is based on the selective amplification of restriction fragments obtained from the digestion of total genomic DNA. As they do not require any prior knowledge about the genome, a large number of markers are easily and quickly available. The resulting markers are dominant (heterozygotes cannot be distinguished from dominant homozygotes) and biallelic (for a given size, the fragment is either present or absent). On the other hand, microsatellites consist of tandemly repeated units of short nucleotide motifs, 1-6 bp long, and they show extensive length variation between individuals (Jarne and Lagoda, 1996). Unlike AFLPs, they often require a costly and time-consuming period of library construction and prescreening before appropriate primers are designed. This is especially true for plant species, as microsatellites are five times less abundant in plants than in mammals (Lagercranz et al, 1993). However, microsatellites present the advantage of being codominant and multiallelic. Thus, two extreme strategies can be adopted when choosing a molecular technique for a genetic diversity assessment: (i) sampling numerous poorly informative markers (AFLPs) or (ii) sampling few highly informative markers (microsatellites).

Numerous studies considered the problem of data analysis for AFLP and other dominant markers. These 
studies have described the variance and potential bias of the diversity and differentiation estimators obtained from dominant markers, and developed appropriate statistical procedures for the analysis of such data. They all showed that dominance and biallelic state of markers increase the variance of estimators, and, in some cases, may lead to significant biases (Lynch and Milligan, 1994; Isabel et al, 1999; Jorde et al, 1999; Krutovskii et al, 1999; Zhivotovsky, 1999; Krauss, 2000). Moreover, expected heterozygosity $(\mathrm{He})$ measured by dominant markers is necessarily bound between 0 and 0.5 , reaching a maximum value when band absence and band presence have equal frequencies $(p=0.5)$. Finally, different markers are characterized by different mutation rates: it is estimated that $10^{-3}-10^{-4}$ mutations per locus per generation occur for microsatellites (Estoup and Angers, 1998; Mariette et al, 2001), whereas the mutation rate for AFLP is about $10^{-6} /$ locus/generation (Mariette et al, 2001). As a consequence, absolute values of diversity and differentiation calculated on data from different types of markers, especially from dominant and codominant markers, should not be directly compared. Owing to the intrinsic differences cited above (different numbers of alleles and mutation rates), within-population diversity estimated with microsatellites should be higher than with AFLPs. On the contrary, because differentiation indices, such as $F_{\text {st }}$, are calculated as ratios of amongover within-population genetic variance (Weir and Cockerham, 1984), differentiation is expected to be lower when assessed through microsatellites compared to more slowly evolving markers (Hedrick, 1999; Balloux et al, 2000). This is a consequence of both the higher number of alleles of microsatellites and their higher probability of homoplasy (see Discussion). Thus, instead of comparing absolute diversity and differentiation values estimated with different types of markers, only global qualitative patterns should be compared, such as the ranking of populations for genetic diversity or differentiation, or the agreement (or not) of the data with a given biological model (eg isolation by distance).

Empirical studies describing diversity and differentiation measures obtained from different markers are abundant in literature, but comparisons between AFLP and microsatellite markers are still rarely found (reviewed in Mariette, 2001). Most comparisons were undertaken to identify and classify distinct cultivars/ varieties of crop species involved in breeding programs. The results obtained by these studies were rather contradictory. Crouch et al (1999), working on Musa breeding populations, found a poor correlation between estimates of genetic distance based on random amplified fragment DNA (RAPDs), AFLPs and microsatellites. In contrast, Roa et al (2000) reported significant Mantel tests between AFLP and microsatellite genetic similarities calculated across seven species of the Manihot genus. Teulat et al (2000) and Pejic et al (1998) also reported congruent patterns of genetic distances between Cocos nucifera populations and maize inbred lines, respectively. As proposed by Powell et al (1996), the strength of the correlation between genetic distances calculated from AFLPs and microsatellites could increase when the among-taxa genetic divergences themselves increase. Indeed, they found that RFLP, AFLP, RAPD and microsatellite genetic similarities were more closely related when calculated between species of the Glycine genus than when calculated within species. Adopting population-based approaches, Mariette et al (2001) working on Pinus pinaster (2001) and Quercus sp. (2002a) and Maguire et al (2002) - working on Avicennia marina found that global differentiation patterns obtained via AFLP and microsatellite markers were congruent. Correlations between diversity estimates were low, except when performed at the regional or subspecific levels rather than at the population level (Mariette et al, 2001, 2002a). If we also consider RAPDs vs microsatellites comparisons, Sun et al (1998) reported a significantly positive correlation between diversity levels obtained with both markers within Elymus fibrosus populations, whereas in their study on Pinus contorta, Thomas et al (1999) found a positive but nonsignificant correlation (recalculated by Mariette, 2001). Concerning estimates of population differentiation, Cagigas et al (1999), on Salmo trutta, and Huang et al (2000), on Haliotis rubra, found that RAPD and microsatellites were in agreement.

Eryngium alpinum L. is a perennial plant found across the European Alps. Owing to changes in land use (disappearance of late hay harvest and extension of early grazing), this species is declining over its entire distribution area and is protected throughout Europe (European Habitat Directive; Wyse Jackson and Akeroyd, 1994). A previous AFLP assessment of genetic diversity in French populations of E. alpinum (Gaudeul et al, 2000) showed that within-population diversity was rather high compared to other AFLP studies. Populations were highly differentiated between, but also within, Alpine valleys, and gene flow appeared extremely low. Here, we report the microsatellite analysis of 12 of the French populations previously studied with AFLPs (Gaudeul et al, 2000). Our aims were: (i) to complete our previous AFLP survey by estimating the within-population fixation index $\left(F_{\text {is }}\right)$ with codominant markers; (ii) to compare estimates of population diversity and differentiation obtained via both markers through nonparametric rank correlations and multivariate analyses; and (iii) to compare the utility of AFLPs and microsatellites for assigning individuals to their population of origin. Assignment tests are increasingly used on codominant data, either for studying migration patterns among demes or to detect illegal picking or poaching of protected species (Manel et al, 2002). Assignment tests have been previously computed on dominant markers (Haig et al, 1997; Lougheed et al, 2000), but, to our knowledge, only one specific program is available yet (Duchesne and Bernatchez, 2002), which does not allow for missing data. Thus, we aimed to develop an appropriate and convenient program to compute assignment tests using dominant markers. The only comparison of codominant and dominant markers on the basis of their relative assignment power was undertaken considering six microsatellite markers and 15 RAPDs (Lougheed et al, 2000). In this case, the authors found evidence that microsatellites performed better than RAPDs. However, dominant markers are usually much more numerous, and we may expect that the loss of information encountered with AFLP or RAPD markers as a result of dominance would be balanced by a high number of polymorphic loci. This was proved on parentage assignment (Gerber et al, 2000) and in the estimation of male mating success (Milligan and McMurray, 1993). 
This kind of empirical, comparative analysis should provide information on the relative information content of different markers such as microsatellites and AFLPs, and should help elucidate effective sampling strategies in genetic diversity studies using molecular markers.

\section{Materials and methods}

\section{Plant material}

E. alpinum L. is a diploid $(2 n=16)$, herbaceous perennial with longevity probably greater than $15-20$ years (M Chaud, personal communication). The species can be found throughout the European Alps (Cherel and Lavagne, 1982), at altitudes between 1200 and $2100 \mathrm{~m}$, where it grows in open habitats such as hayfields or avalanche corridors.

The taproot persists over winter whereas the aerial stems and leaves, measuring $50 \mathrm{~cm}$ to $1 \mathrm{~m}$ high, disappear each year. Each flowering individual generally produces one to five stems, each of them bearing one to five inflorescences. Each inflorescence produces 200-300 small, white flowers, composed of two ovules and five stamens. Field observations (unpublished data) showed that E. alpinum is mainly pollinated by opportunistic insects such as honeybees and bumblebees. Selfing appears possible, but partial self-incompatibility and/ or early-acting inbreeding depression are suspected (unpublished data). Mature fruits (schizocarpous diachenes) fall near the mother plant at the end of August. The dried sepals of these fruits may attach to the fur of animals and favor secondary dispersal.
Twelve French populations of E. alpinum, chosen to cover a broad geographic range (Figure 1), were sampled during the summer of 1998 . The population sizes are widely distributed, ranging from fewer than hundred to hundreds of thousands individuals. In each population, leaf material from 24 widespread individuals was collected, dried in silica gel and stored at room temperature. In Ecrins and Vanoise, three (EE05, EE06 and EE10) and two (EV01 and EV02) populations, respectively, were very close $(400 \mathrm{~m}$ to $8 \mathrm{~km}$ apart). Moreover, in five populations (EE05, EE06, EE10, EV02 and EO03), plants were sampled in different parts of the populations $(3,3,3,2$ and 2 subpopulations, respectively).

\section{AFLP procedure}

The precise AFLP protocol was detailed in Gaudeul et al (2000). Total genomic DNA was extracted from leaf material using the 'DNeasy Plant Mini Kit' (QIAGEN) following the manufacturer's protocol. After digestion with EcoR1 and Mse1, adaptors were ligated on both ends of genomic fragments and a two-step selective amplification was performed. We chose three selective primer pairs: EcoR1.AGA/Mse1.CAGG, EcoR1.ATC/ Mse1.CTCT and EcoR1.ATC/Mse1.CTCG (only the selective bases are indicated). Resulting PCR products were loaded on 5\% Long Ranger polyacrylamide gels, and electrophoresis was run for $6 \mathrm{~h}$ on an ABI 377 automated sequencer (Perkin-Elmer). AFLP patterns were then visualized with GeneScan Analysis ${ }^{\circledR} 3.1$ (Applied Biosystems), and 63 independent markers were typed as present or absent.

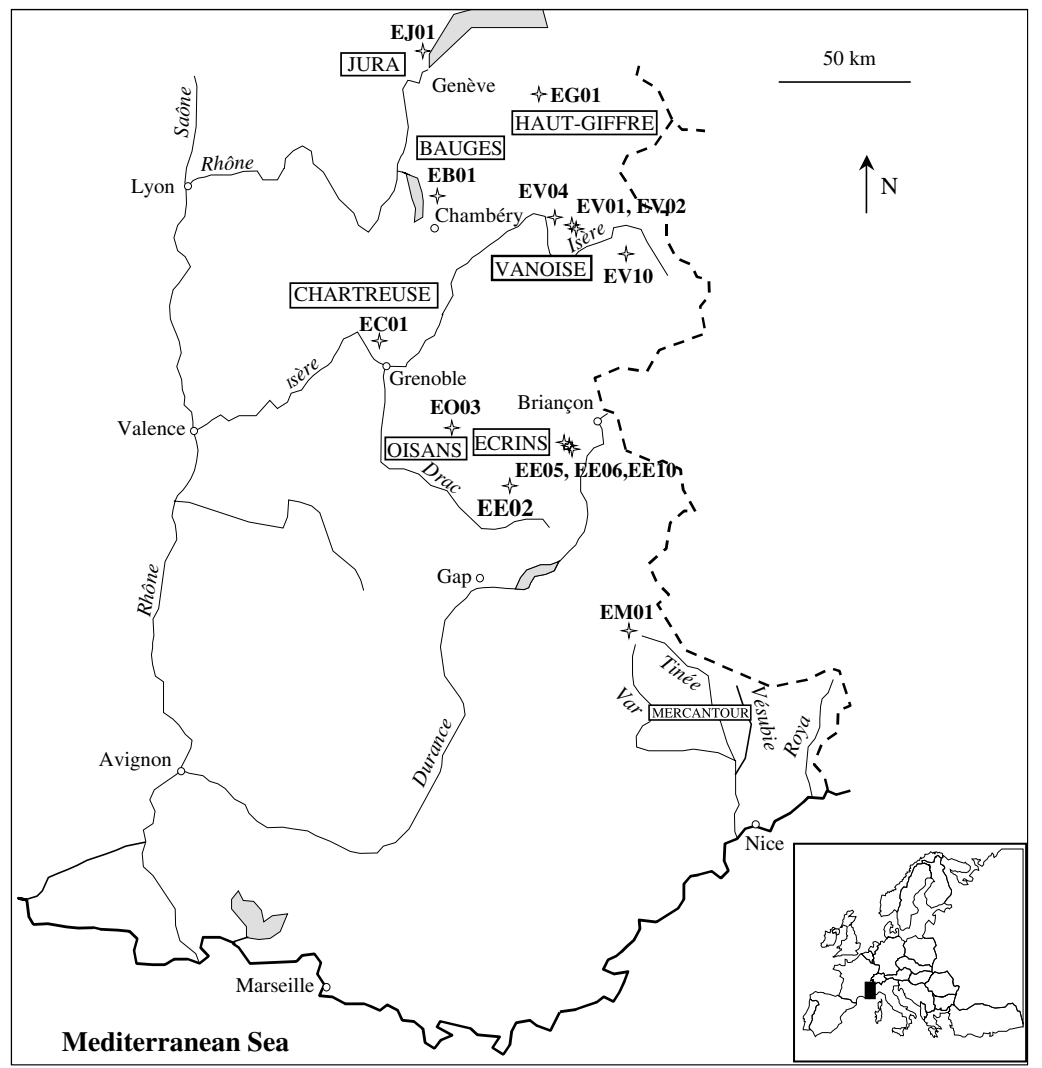

Figure 1 Geographic location of the 12 studied populations of E. alpinum L. The inset shows the position of the studied area in France. 
Table 1 Within-population diversity indices calculated from microsatellite and AFLP data

\begin{tabular}{|c|c|c|c|c|c|c|}
\hline \multirow[t]{2}{*}{ Population } & \multirow[t]{2}{*}{ Approximate size } & \multicolumn{3}{|c|}{ Microsatellite-based estimates } & \multicolumn{2}{|c|}{ AFLP-based estimates } \\
\hline & & Mean allelic richness over loci & Unbiased He & $\mathrm{F}_{i s}$ & $\%$ polymorphic markers & Nei index \\
\hline EJ01 & 1000 & 2.09 & 0.244 & -0.088 (NS) & 52.4 & 0.206 \\
\hline EB01 & 750 & 2.61 & 0.388 & -0.125 (NS) & 39.7 & 0.167 \\
\hline EV01 & 500 & 2.96 & 0.484 & -0.028 (NS) & 55.6 & 0.201 \\
\hline EV02 & 500 & 2.77 & 0.460 & 0.030 (NS) & 57.1 & 0.215 \\
\hline EV04 & 100 & 2.75 & 0.417 & -0.106 (NS) & 54.0 & 0.185 \\
\hline EV10 & 100 & 3.40 & 0.471 & 0.007 (NS) & 50.8 & 0.206 \\
\hline EO03 & 1000 & 3.05 & 0.480 & -0.056 (NS) & 57.1 & 0.221 \\
\hline EE02 & 35 & 1.94 & 0.348 & -0.193 (NS) & 39.7 & 0.134 \\
\hline EE05 & 5000 & 2.59 & 0.425 & 0.092 (NS) & 65.1 & 0.234 \\
\hline EE06 & 750 & 2.89 & 0.404 & 0.107 (NS) & 55.6 & 0.196 \\
\hline EE10 & 200000 & 2.79 & 0.418 & $-0.070(\mathrm{NS})$ & 60.3 & 0.212 \\
\hline $\mathrm{EM}^{\mathrm{a}}$ & 100000 & 3.30 & 0.324 & 0.055 (NS) & 65.1 & 0.221 \\
\hline
\end{tabular}

For each population, the first letter (E) stands for E. alpinum, the second one is the initial of the mountain range where the population was sampled ('J' is for Jura, ' $\mathrm{B}$ ' for Bauges, ' $\mathrm{V}$ ' for Vanoise, ' $\mathrm{O}$ ' for Oisans, ' $\mathrm{E}$ ' for Ecrins and ' $\mathrm{M}$ ' for Mercantour), and the number denotes different populations from the same range. NS= nonsignificant.

aithout microsatellite locus Ealp035.

\section{Microsatellite procedure}

Samples were genotyped at seven microsatellite loci described in Gaudeul et al (2002). Microsatellite PCRs were processed using fluorescent-labeled primers, allowing PCR products to be simultaneously analyzed on an ABI 377 automated sequencer (Perkin-Elmer). Subsequent analysis was carried out using GeneScan Analysis $^{\mathbb{R}} 3.1$ and Genotyper ${ }^{\mathbb{R}} 2.0$ (Applied Biosystems).

\section{Statistical analysis}

The statistical analyses of AFLP data were performed with the software TFPGA (Miller, 1997) and described in Gaudeul et al (2000). They are not fully detailed here, unless additional analyses have been carried out. Unless otherwise stated, statistical analyses of microsatellite data were computed using FSTAT version 2.9.3 (Goudet, 1995), applying sequential Bonferroni-type correction each time multiple tests were involved. For all statistical tests, we chose a significance level $\alpha=0.05$.

Independence of markers: For AFLPs, we calculated a basic similarity index between all pairs of markers on the overall data (Gaudeul et al, 2000), and we discarded markers that were more than $95 \%$ or less than $5 \%$ similar to another one. For microsatellites, genotypic linkage disequilibrium was tested for each pair of loci within each population using the log-likelihood ratio G-statistic.

Within-population genetic diversity: First, in order to compare the discrimination power of each type of marker, we looked for identical multilocus genotypes in each data sets. Then, for AFLP data, we calculated (i) the number of polymorphic bands and (ii) Nei's unbiased expected heterozygosity (Nei, 1987). For microsatellites, we computed (i) allelic richness (a measure of the number of alleles independent of sample size; El Mousadik and Petit, 1996), (ii) expected heterozygosity (He), and (iii) the fixation index, $F_{\text {is }}$ (Weir and Cockerham, 1984). The significance of $F_{\text {is }}$ was assessed by random permutations of alleles in each population at each locus.

Spearman's rank correlations (Lehmann and D'Abrera, 1998) were tested between all pairs of diversity indices and between diversity indices and population size. Kruskall-Wallis tests (Lehmann and D'Abrera, 1998) were carried out in order to test for a significant difference of genetic diversity among populations (based on interlocus variance). Both analyses were performed with MINITAB $^{\circledR}$ version 12.2 .

Genetic differentiation: F-statistics were calculated for both type of markers, adopting the Weir and Cockerham procedure (1984). The unbiased $F_{\mathrm{st}}$ estimator, $\theta$, was calculated over all populations, and jackknifing over loci provided standard deviations. $F_{\text {st }}$ was also estimated between all pairs of populations and between subpopulations within a population when possible. Significance was assessed by performing exact tests of population differentiation (Raymond and Rousset, 1995). For AFLP, allelic frequencies were calculated assuming Hardy-Weinberg equilibrium within populations. This hypothesis was a posteriori justified by null fixation indices $\left(F_{\text {is }}\right)$ estimated from microsatellite data within all populations.

In order to compare geographic distances and genetic distances obtained between populations with both markers, Mantel tests were performed between pairwise $\theta$ values estimated from AFLPs and microsatellites, and between $\theta$ and geographic distances. We also computed Mantel tests on AFLP- and microsatellite-based betweenindividual distances on the overall sampling design and within each of the 12 populations (between-individual distances were calculated with GenAlEx 5.0 and are defined below). Principal coordinate analyses (PCoA) were computed on each data set separately, using GenAlEx 5.0 (Peakall and Smouse, 2001). For a single locus analysis of a microsatellite marker, with $i, j, k$ and $l$ representing alleles of the two individuals, the distance between diploid individuals was defined as $d^{2}(i i, i i)=0$, $d^{2}(i j, i j)=0, d^{2}(i i, i j)=1, d^{2}(i j, i k)=1, d^{2}(i j, k l)=2, d^{2}(i i, j k)=3$ and $d^{2}(i i, j j)=4$ (for more details, see Peakall et al, 1995). For the AFLP data set, the calculation of distances followed the method of Huff et al (1993). The distance was equivalent to the proportion of band differences among two DNA profiles: $D=n\left(1-2 n_{x y} / 2 n\right)$, where $2 n_{x y}$ is the number of shared bands between the two 
individuals $x$ and $y$, and $n$ equals the total number of band positions. This distance was not corrected for sample sizes because they remain roughly constant over populations (18-24 individuals per population). We first considered all the populations. Then, we compared the power of AFLPs and microsatellites for discriminating: (i) individuals of neighboring populations (EE05, EE06, EE10 and EV01, EV02) and (ii) individuals of different subpopulations within a single population (EE05, EE06, EE10, EV02 and EO03).

Assignment tests: Individuals who were genotyped for only three microsatellite loci or less were discarded from each data set in order not to bias the comparison of the two types of markers. Thus, assignment tests were carried out on 240 individuals. To analyze microsatellite data, we adopted the exclusion test of Cornuet et al (1999) based on the approach developed by Paetkau et al (1995). This approach implies that populations are at Hardy-Weinberg equilibrium and that there is no linkage disequilibrium between loci. Briefly, the method uses the allele frequencies from a population sample to compute the likelihood that a given genotype occurs in this population. The significance of the likelihood value is determined in comparison with a distribution of likelihoods of 1000 simulated genotypes for each candidate population. The analysis of AFLP markers was very similar, but band frequencies, instead of allele frequencies, were derived in each population. As a consequence, only the assumption of linkage equilibrium is required (allowing the generalization to more than one loci). As our purpose is to estimate the accuracy provided by AFLPs and microsatellites, we calculated the percentage of individuals correctly assigned to their population of origin using each type of markers. For this, we adopted a leave-one-out method: each individual was removed from the data set, allele or band frequencies were recomputed and the individual was assigned to a population at a $5 \%$ significant level as described above.

In order to determine the optimal number of AFLP markers needed for assigning individuals to their population of origin, we performed assignment tests using a variable number of markers $(n=10,20,30,40$, 50). In each case, $n$ markers were randomly chosen, used for assignment, and this process was repeated 100 times. We computed the mean and the standard deviation of the percentage of correct assignments over the 100 repetitions, obtaining a mean percentage of correct assignment for each number of markers. Then, we tested if these percentages were significantly different using multiple comparisons of means (pairwise Bonferroni method; SAS Institute Inc., 1990).

All the assignment tests used in this paper have been implemented in $\mathrm{C}++$. The code developed to perform assignment tests from AFLP data is available upon request to stephanie.manel@ujf-grenoble.fr.

\section{Results}

\section{Within-population genetic diversity}

No linkage disequilibrium was detected between microsatellite markers. Over all samples and loci, we found a total of 48 alleles. The number of alleles per locus spanned from 2 (Ealp245) to 18 (Ealp1349b), with an average of $6.9 \pm 4.9$ (S.D.) alleles per locus. Locus Ealp035 failed to amplify on individuals from EM01, probably due to mutations and/or indels in the flanking region. Considering microsatellite allele frequencies, we observed that a lot of alleles (23 out of 48) were present in only one to three populations, and, conversely, that alleles that were frequent in most populations were sometimes absent in others, especially in populations EE02, EV01 and EM01 (each of them lacks three alleles observed in at least 10 populations). The same observation holds for AFLP data, but for different populations: EJ01 and EM01 exhibited bands that were absent in all other populations, and EE02 and EV02 lack bands that were found in all other populations. With AFLP data, we found three pairs of identical multilocus genotypes; all of them corresponded to individuals within populations (one pair in EV10 and two pairs in EV04). With microsatellite data, one genotype was found five times, three were found three times and nine were found two times. In two cases, these identities involved individuals from two distinct populations (EE02-EO03 and EE05EE06).

Rank correlations were significantly positive among AFLP diversity indices, between AFLP indices and population size, and between AFLP indices and $F_{\text {is }}$ calculated from microsatellites (Table 2). All the other correlations were nonsignificant. Kruskall-Wallis tests indicated that diversity measures were not significantly different among populations, neither with AFLP (Nei's index, $P=0.42$ ) nor with microsatellite data (allelic richness, $P=0.39$; unbiased $\mathrm{He}, P=0.80$; fixation index, $P=0.49)$. Within-population fixation indices, $F_{\text {is, }}$ were never significantly different from zero (Table 1).

\section{Genetic differentiation}

For the 12 populations studied, we obtained $\theta_{\text {microsat. }}=0.23 \pm 0.05$. Between all pairs of populations, $\theta_{\text {microsat. }}$ values spanned from 0.005 (between EE05 and EE06) to 0.48 (between EM01 and EE02; Table 3). Exact tests of population differentiation were significant in all cases $(0.01<P<0.05)$ except between close populations (EE05-EE06-EE10 and EV01-EV02) and between EE02 and EE10 $(P>0.05)$. AFLP data lead to an overall $\theta_{\mathrm{AFLP}}=0.42 \pm 0.04$. Between pairs of populations, mean $\theta_{\mathrm{AFLP}}=0.41 \pm 0.10$, with extreme values of $\theta_{\mathrm{AFLP}}=0.21$ between EE05 and EVO4, and $\theta_{\mathrm{AFLP}}=0.63$ between EB01 and EE02. Differentiation tests were all highly significant, even between close populations (all $P<0.001$ except for EE06-EE10: $0.001<P<0.01$; Gaudeul et al, 2000). When performed between subpopulations within populations, differentiation tests were not significant, neither with microsatellite nor with AFLP data. Mean $\theta$ values between pairs of subpopulations were 0.11 with AFLPs and 0.02 with microsatellites.

Mantel tests revealed that between-population genetic distances $(\theta)$ calculated from AFLPs were significantly correlated with those calculated from microsatellite data $(r=0.50, P<0.001)$. Both genetic distances were also correlated with geographic distances between pairs of populations $\left(r_{\text {microsat. }}=0.61, P<0.001\right.$, and $r_{\mathrm{AFLP}}=0.28$, $P=0.02)$. On the overall sampling design, betweenindividual genetic distances calculated from AFLP and microsatellite data were significantly correlated 
Table 2 Spearman's rank correlation between diversity indices and size of populations

\begin{tabular}{|c|c|c|c|c|c|c|}
\hline & \multirow[t]{2}{*}{ Population size } & \multicolumn{3}{|c|}{ Microsatellite-based estimates } & \multicolumn{2}{|c|}{ AFLP-based estimates } \\
\hline & & Mean allelic richness & Unbiased He & $\mathrm{F}_{i s}$ & $\%$ polymorphic markers & Nei index \\
\hline \multirow[t]{2}{*}{ Mean allelic richness } & 0.300 & - & & & & \\
\hline & 0.344 & & & & & \\
\hline \multirow[t]{2}{*}{ Unbiased He } & 0.148 & -0.158 & - & & & \\
\hline & 0.646 & 0.625 & & & & \\
\hline \multirow{2}{*}{$F_{\text {is }}$} & 0.433 & -0.217 & 0.28 & - & & \\
\hline & 0.160 & 0.498 & 0.379 & & & \\
\hline \multirow[t]{2}{*}{$\%$ polym. markers } & 0.722 & 0.044 & 0.039 & 0.616 & - & \\
\hline & $0.008^{* *}$ & 0.892 & 0.905 & $0.033^{*}$ & & \\
\hline \multirow[t]{2}{*}{ Nei index } & 0.677 & 0.142 & 0.418 & 0.656 & 0.855 & - \\
\hline & $0.016^{*}$ & 0.659 & 0.177 & $0.020^{*}$ & $<0.001^{* * *}$ & \\
\hline
\end{tabular}

Upper lines: correlation coefficient $(r)$; lower lines: exclusion probability $(P)$. When the correlation is significant, $* 0.01<P<0.01$, ${ }^{* *} 0.001<P<0.01$ and ${ }^{* * *} P<0.001$.

Table 3 Genetic differentiation between all pairs of populations obtained from microsatellite data

\begin{tabular}{|c|c|c|c|c|c|c|c|c|c|c|c|}
\hline & EJ01 & $E B 01$ & EV01 & EV02 & EV04 & EV10 & EO03 & EE02 & EE05 & EE06 & EE10 \\
\hline EJ01 & - & & & & & & & & & & \\
\hline EB01 & $\underset{*}{0.347}$ & - & & & & & & & & & \\
\hline EV01 & $\underset{*}{0.341}$ & $\underset{*}{0.288}$ & - & & & & & & & & \\
\hline EV02 & $\begin{array}{c}0.384 \\
*\end{array}$ & $\begin{array}{c}0.280 \\
*\end{array}$ & $\begin{array}{c}0.048 \\
\text { NS }\end{array}$ & - & & & & & & & \\
\hline EV04 & $\begin{array}{c}0.399 \\
*\end{array}$ & $\begin{array}{c}0.283 \\
*\end{array}$ & $\begin{array}{c}0.164 \\
*\end{array}$ & $\underset{*}{0.102}$ & - & & & & & & \\
\hline EV10 & $\begin{array}{c}0.405 \\
*\end{array}$ & $\underset{*}{0.163}$ & $\underset{*}{0.182}$ & $\underset{*}{0.102}$ & $\underset{*}{0.127}$ & - & & & & & \\
\hline EO03 & $\underset{*}{0.291}$ & $\underset{*}{0.215}$ & $\underset{*}{0.114}$ & $\underset{*}{0.095}$ & $\underset{*}{0.097}$ & $\underset{*}{0.090}$ & - & & & & \\
\hline EE02 & $\begin{array}{c}0.424 \\
*\end{array}$ & $\begin{array}{c}0.387 \\
*\end{array}$ & $\begin{array}{c}0.175 \\
*\end{array}$ & $\underset{*}{0.150}$ & $\underset{*}{0.232}$ & $\begin{array}{c}0.229 \\
*\end{array}$ & $\begin{array}{c}0.095 \\
*\end{array}$ & - & & & \\
\hline EE05 & $\begin{array}{c}0.322 \\
*\end{array}$ & $\underset{*}{0.275}$ & $\underset{*}{0.170}$ & $\begin{array}{c}0.089 \\
*\end{array}$ & $\underset{*}{0.143}$ & $\underset{*}{0.143}$ & $\underset{*}{0.086}$ & $\underset{*}{0.126}$ & - & & \\
\hline EE06 & $\underset{*}{0.301}$ & $\begin{array}{c}0.239 \\
*\end{array}$ & $\underset{*}{0.200}$ & $\underset{*}{0.124}$ & $\underset{*}{0.145}$ & $\underset{*}{0.144}$ & $\underset{*}{0.080}$ & $\underset{*}{0.154}$ & $\begin{array}{c}0.005 \\
\text { NS }\end{array}$ & - & \\
\hline EE10 & $\underset{*}{0.324}$ & $\underset{*}{0.275}$ & $\underset{*}{0.191}$ & $\underset{*}{0.117}$ & $\underset{*}{0.178}$ & $\underset{*}{0.160}$ & $\underset{*}{0.096}$ & $\begin{array}{c}0.120 \\
\text { NS }\end{array}$ & $\begin{array}{c}0.019 \\
\text { NS }\end{array}$ & $\begin{array}{c}0.007 \\
\text { NS }\end{array}$ & - \\
\hline EM01 & $\begin{array}{c}0.322 \\
*\end{array}$ & $\begin{array}{c}0.364 \\
*\end{array}$ & $\begin{array}{c}0.447 \\
*\end{array}$ & $\begin{array}{c}0.467 \\
*\end{array}$ & $\begin{array}{c}0.447 \\
*\end{array}$ & $\underset{*}{0.379}$ & $\begin{array}{c}0.333 \\
*\end{array}$ & $\begin{array}{c}0.477 \\
*\end{array}$ & $\begin{array}{c}0.423 \\
*\end{array}$ & $\underset{*}{0.403}$ & $\underset{*}{0.418}$ \\
\hline
\end{tabular}

The first line contains $F_{\mathrm{st}}$ estimates, the second line indicates the significance levels of exact tests of population differentiation (Raymond and Rousset, 1995). NS = nonsignificant, ${ }^{*} 0.01<P<0.05$.

$(P<0.001)$. However, within populations, the correlation was significant in only two populations out of 12 (EJ01, $P=0.01 ; \mathrm{EV} 02, P=0.01)$.

PCoA provided visual representations of genetic proximities between all populations, between close populations and between subpopulations within- population. The variance explained by the first two axes was always greater for microsatellites than for AFLPs (eg 66 vs $50 \%$ when considering all populations). In both cases, most populations were rather clustered and a few others seemed more distant (namely EJ01, EB01 and EM01 for microsatellite data, and EJ01, EM01, EE02 and EV02 for AFLP data; Figure 2). These populations were also apart from the others along the third axis of the PCA (not shown). Populations from the same mountain range (EE02, EE05, EE06, EE10 from Ecrins, and EV01, EV02, EV04, EV10 from Vanoise) appeared closer with microsatellite than with AFLP data. When considering only close populations (Figure 3), it is obvious that AFLPs can differentiate individuals from neighboring populations, whereas microsatellites do not offer this opportunity. Indeed, with microsatellites, individuals of distinct populations were intermingled and no overall pattern emerged. Last, within populations, neither AFLP nor microsatellites managed to distinguish individuals from distinct subpopulations (results not shown).

\section{Assignment tests}

Considering the overall data set, 90.8 and $77.1 \%$ of individuals were correctly assigned to their population of origin with AFLP and with microsatellite data, respectively. When we restricted the analysis to neighboring populations, the effectiveness of AFLP markers did not decrease (correct assignments reached 92.7 and 92.9\% in Fournel and Pralognan, respectively). In 


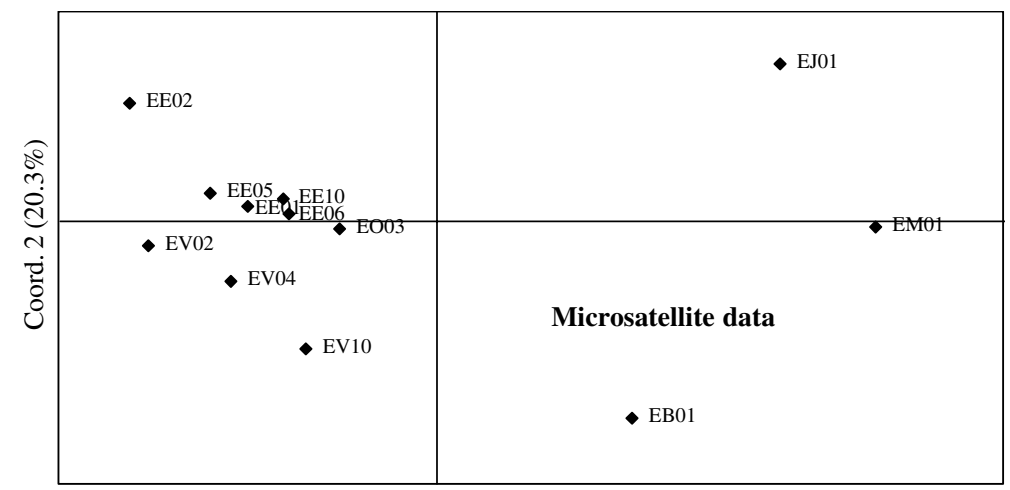

Coord. $1(45.7 \%)$

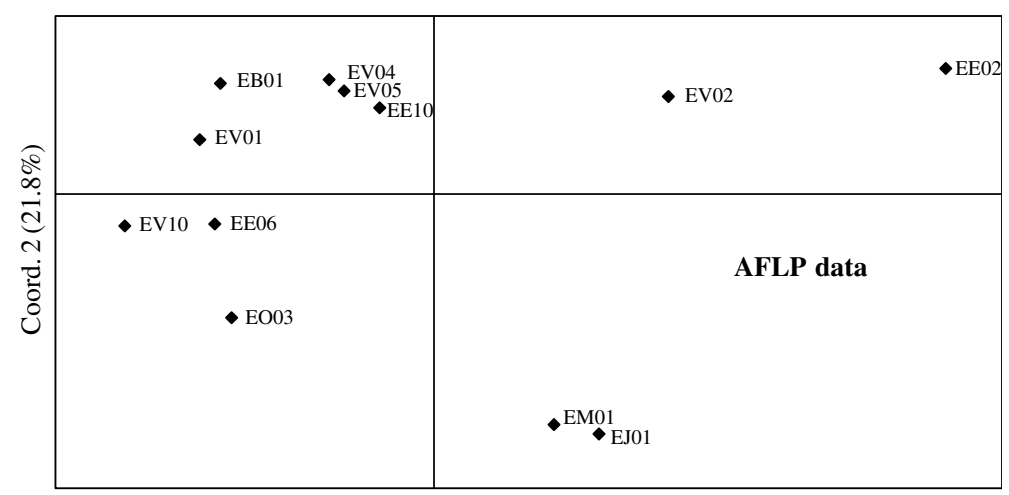

Coord. $1(28.3 \%)$

Figure 2 PCoA computed on the 12 populations, on AFLP and microsatellite data. Percentages of total variance explained by each axis are noted in brackets.
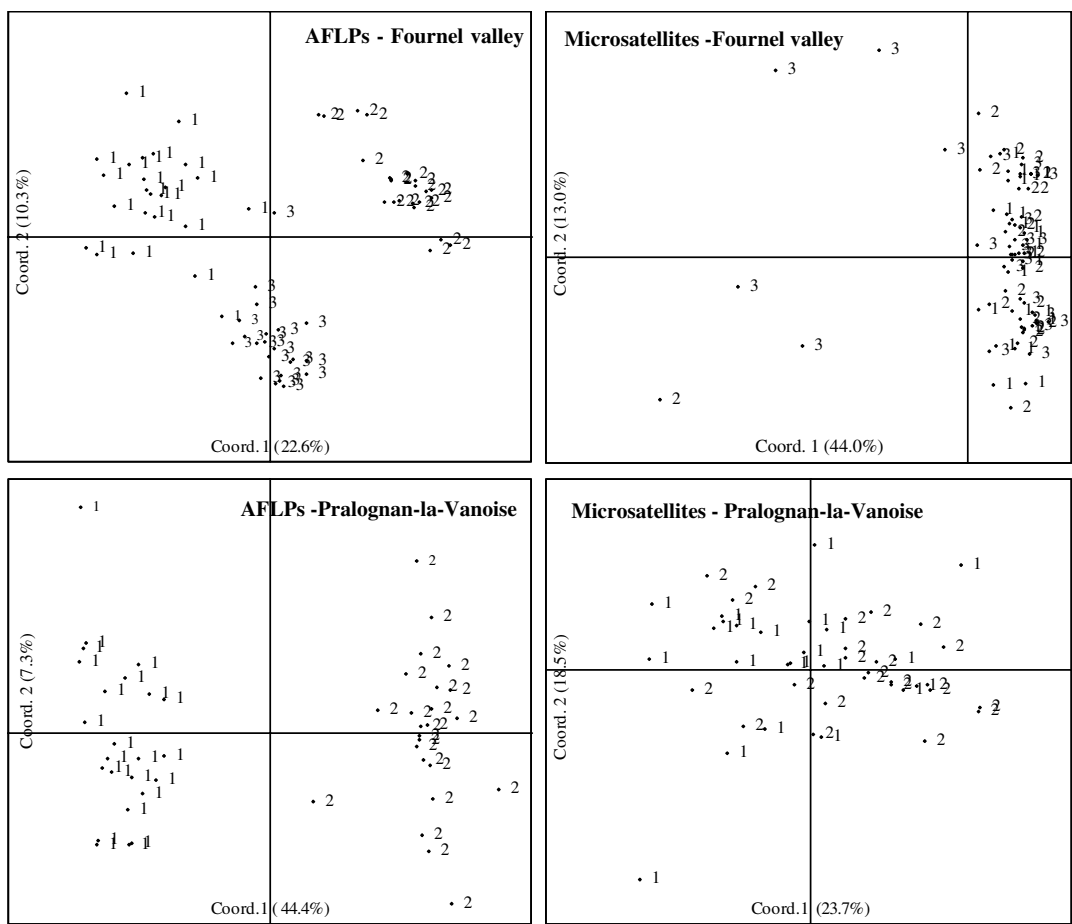

Figure 3 PCoA computed on close populations from the Fournel valley and from Pralognan-la-Vanoise, on AFLP and microsatellite data. For the Fournel valley, individuals of EE05, EE06 and EE10 are noted 1, 2 and 3, respectively. For Pralognan-la-Vanoise, individuals from EV01 and EV02 are numbered 1 and 2, respectively. Percentages of total variance explained by each axis are noted in brackets. 


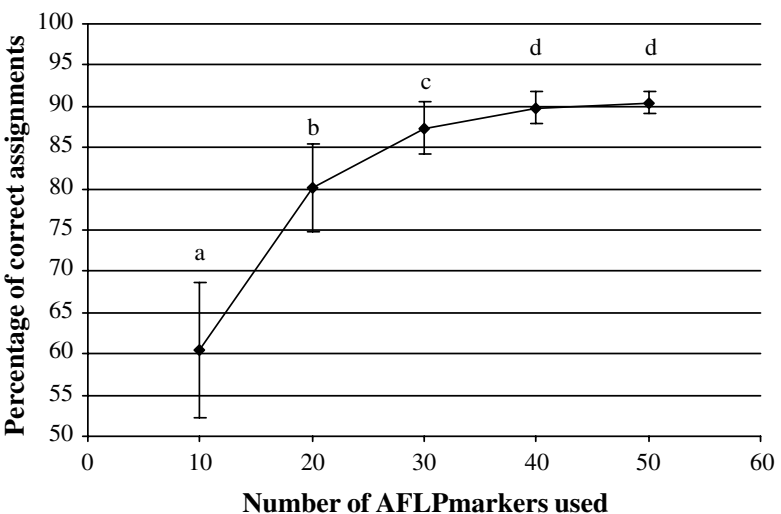

Figure 4 Relationship between the percentage of individuals correctly assigned to their population of origin and the number of AFLP markers used. Different letters indicate significantly different values (multiple comparisons of means, $P<0.05$ ).

contrast, the values obtained with microsatellite data dropped to 50.9 and $69.0 \%$. The percentage of correct assignments increased with the number of AFLP markers when this number was comprised between 10 and 40 , but the assignment success was not different when 40 or 50 markers were considered (89.9 and $90.4 \%$, respectively; Figure 4). Thus, with our data, 40 AFLP markers seem to be enough to correctly assign individuals to their population of origin.

\section{Discussion}

The aim of this study was to compare the performance of two types of molecular markers (microsatellites and AFLPs) in the assessment of genetic diversity and differentiation in E. alpinum L.

The ranking of populations based on their diversity indices was not correlated between AFLP and microsatellite data sets, except for the fixation index $\left(F_{\text {is }}\right)$, which was positively linked to the two AFLP indices. Moreover, as expected from population genetics theory, we found that AFLP diversity increased significantly with the size of populations, whereas no such trend was observed on microsatellite data. Such discrepancies between different kinds of molecular markers are often observed in comparative studies (see Introduction and references therein). They can be explained by: (i) the number of markers used, (ii) the relative mutation rates of the different markers, and (iii) the level of population heterogeneity in diversity levels. First, the small number of microsatellite loci could be involved. Indeed, seven markers are probably not enough to provide an exact view of the whole genome. Using computer simulations, Mariette et al (2002b) showed that low gene flow induced high differences in variation levels among loci (referred to as 'genomic heterogeneity'), thus making even more important to consider many loci. In this case, AFLPs, in spite of their dominance, were more reliable than microsatellites as soon as they were at least four times more numerous. Here, differentiation is high, and we compared patterns obtained with 63 AFLP markers to those deduced from seven microsatellites. Thus, in this study, AFLPs are probably better suited for estimating genetic diversity than a few microsatellites due to their broader coverage of the genome. Another plausible explanation for the lack of relationship between population size and diversity at microsatellite loci is a much higher mutation rate at these loci: although diversity is expected to be related to the product of effective population size and mutation rate, populations may approach asymptotic levels of diversity very quickly as a function of their size. This is due to the saturation of the markers beyond a certain level of polymorphism, also called homoplasy. Finally, the differences in levels of diversity were nonsignificant and the observed ranking of populations could thus only be generated by the random variation of diversity, leading to the lack of correlation between diversity measures calculated from different markers (Mariette et al, 2002b).

Considering population differentiation, estimates of $F_{\text {st }}$ were always higher when calculated from AFLP than from microsatellite data. The number of markers does not strongly influence these estimates (Mariette, 2001). As explained before (see Introduction), lower differentiation measures are expected with microsatellites because they have higher mutation rates and estimates of withinpopulation genetic diversity. Thus, mutation is not negligible compared to migration and drift. In addition, high levels of polymorphism may also lead to significant homoplasy, further decreasing genetic divergence between populations. Moreover, Isabel et al (1999) and Krutovskii et al (1999) demonstrated that the dominance of AFLP markers leads to an upwardly biased differentiation index, especially when sampling within populations was low (less than 30 individuals). Here, we found global differentiation indices of $\theta=0.40$ with AFLPs and $\theta=0.23$ with microsatellites. Thus, both data sets conclude that there is very strong differentiation and extremely low gene exchange between populations. The exact $F_{\text {st }}$ value does not matter much: as mutation is probably high compared to migration, $F_{\text {st }}$ estimates cannot be used to infer the effective number of migrants $\left(N_{\mathrm{m}}\right)$. Moreover, Mantel tests performed on betweenpopulation genetic distances obtained by both markers were significant, and PCoA revealed globally congruent patterns of genetic proximities between populations. Nevertheless, it is worth noting that the Mantel test is much more significant between microsatellite and geographic distances $(P<0.001)$ than between AFLP and geographic distances $(P=0.02$; this test was not significant when two distant populations were added, Gaudeul et al, 2000). Again, this result raises the question of the homoplasy of molecular markers. Divergence between identity by state and identity by descent should be considered because it is an important caveat that may cause erroneous conclusions with regard to genetic relationships among populations. Because of their biallelic character, homoplasy is unavoidable in AFLP markers: whereas the presence of a band corresponds to a single DNA sequence, its absence may be due to several nucleotide changes, which cannot be distinguished. Moreover, the homology of comigrating fragments is only very rarely checked when using fingerprinting techniques such as AFLP (but see Thormann et al, 1994; Rieseberg, 1996; Peakall et al, 1998; Hurme and Savolainen, 1999). Homoplasy is also very likely to occur with microsatellite markers, given their high mutation rates and our lack of understanding of their mutation processes (Estoup and Angers, 1998). For both types of markers, homoplasy probably increases 
with genetic divergence between samples, thus precluding their use at large spatial and temporal scales, or between taxonomically divergent samples. Here, the lower correlation between AFLP and geographic distances suggests higher homoplasy of AFLPs compared to microsatellites.

Individual genetic distances obtained from AFLPs and microsatellites were significantly correlated, but this was only due to the interpopulation comparisons. Indeed, within populations, Mantel tests between AFLP and microsatellite distances were no longer significant (except in two cases out of 12). The observation that dominant AFLPs and microsatellites can reveal concordant patterns among populations, but not within populations has been shown for other taxa (Powell et al, 1996; Maguire et al, 2002). This suggests that the two sets of markers are probably segregating independently, but that ecologically and evolutionary important groupings are the same with both sets of markers. If we now consider the relative patterns observed with AFLP and microsatellites on an intermediate spatial scale, important discrepancies also appear: AFLPs suggested significant differentiation between close populations. On the contrary, microsatellites cannot distinguish them, as shown by nonsignificant differentiation tests, by multivariate analysis (PCoA) where all individuals are intermingled, and by assignment tests. AFLPs offer the opportunity for assigning each individual to its population of origin with a relatively constant rate over the range of genetic distance between populations. Microsatellites were as effective as AFLPs for assigning individuals among distant populations, but they were less efficient on the overall dataset and their rate of correct assignment radically decreased on a local scale. This may be associated with the higher polymorphism of these markers. Previous studies have shown that at the lowest hierarchical level, microsatellites always displayed the lowest values of similarity when compared to other markers, reflecting their high information content (Russell et al, 1997, Pejic et al, 1998, Teulat et al, 2000). As a result, they appear less suitable for assigning individuals on this intermediate spatial scale, but microsatellites could be more efficient than AFLPs for investigating within-population structure and are widely preferred over AFLPs for fine-scale genetic studies (eg Streiff et al, 1998; Ueno et al, 2000; Bonnin et al, 2001). For this purpose, their number should, however, be increased in order to reach a higher number of distinct multilocus genotypes.

Research programs are often limited by technical problems, sometimes impeding a biological question to be properly addressed. When available, microsatellites are usually considered the markers of choice for investigating genetic structure, while dominant AFLPs are mostly used when fast and inexpensive surveys are needed. However, the present study provides information on the relative effectiveness of both kinds of markers in estimating population genetic diversity and structure, and suggests that the choice between them depends on the biological question to be addressed. The relative number of markers provided by each technique should also be taken into account. If the main purpose is to evaluate within-population diversity, a high number of dominant markers appear preferable to a few microsatellites, because coverage of the genome will be more complete. However, high levels of polymorphism and codominance found in microsatellites make them more suitable than AFLPs for investigating fine-scale spatial structure within populations. In E. alpinum, global patterns of between-population differentiation obtained with both types of markers were congruent. AFLPs were more efficient than microsatellites in discriminating neighboring populations, and in assigning individuals to their population of origin, but they seem to suffer higher levels of homoplasy when more divergent populations are studied.

Of course, the simultaneous use of numerous kinds of markers (AFLP, microsatellites, allozymes, DNA sequences, etc.) will always be very informative, especially if these markers exhibit various characteristics such as different mutation rates, different segregation patterns (eg nuclear vs cytoplasmic markers) or if they are influenced by distinct evolutionary forces (eg isozymes are more likely to be under selection than microsatellites). Even if such studies are still rare in the evolutionary biology literature (but see Ross et al, 1999), this kind of meta-analysis is being initiated more frequently and is at the origin of a new research area called 'population genomics' (Hedges, 2000; Black et al, 2001). Owing to recent technical and statistical advances, it is likely to represent an important step forward for population biology.

\section{References}

Balloux F, Brünner H, Lugon-Moulin N, Hausser J, Goudet J (2000). Microsatellites can be misleading: an empirical and simulation study. Evolution 54: 1414-1422.

Black WC, Baer CF, Antolin MF, DuTeau NM (2001). Population genomics: genome-wide sampling of insect populations. Annu Rev Entomol 46: 441-469.

Bonnin I, Ronfort J, Wozniak F, Olivieri I (2001). Spatial effects ad rare outcrossing events in Medicago truncatula (Fabaceae). Mol Ecol 10: 1371-1383.

Cagigas ME, Vazquez E, Blanco G, Sanchez JA (1999). Combined assessment of genetic variability in populations of Brown trout (Salmo trutta L.) based on allozymes, microsatellites and RAPD markers. Mar Biotechnol 1: 286-296.

Cherel O, Lavagne A (1982). Aire de répartition, phénologie, biologie, reproduction d'Eryngium alpinum, 'la Reine des Alpes', dans la vallée du Fournel. Propositions de mesures de protection de l'espèce. Trav Sci Parc Natl Ecrins 2: 53-92.

Cornuet JM, Piry S, Luikart G, Estoup A, Solignac M (1999). New methods employing multilocus genotypes to select or exclude populations as origins of individuals. Genetics 153: 1989-2000.

Crouch JH, Crouch HK, Constandt H, Van Gysel A, Breyne P, Van Montagu M et al (1999). Comparison of PCR-based molecular analyses of Musa breeding populations. Mol Breed 5: $233-244$

Duchesne P, Bernatchez L (2002). AFLPOP: a computer program for simulated and real population allocation, based on AFLP data. Mol Ecol Notes 2: 380-383.

El Mousadik A, Petit RJ (1996). High level of genetic differentiation for allelic richness among populations of the Argan tree [Argania spinosa (L.) Skeels] endemic to Morocco. Theor Appl Genet 92: 832-839.

Estoup A, Angers B (1998). Microsatellites and minisatellites for molecular ecology: theoretical and experimental considerations. In: Carvalho G (ed) Advances in Molecular Ecology. NATO Press: Amsterdam.

Gaudeul M, Taberlet P, Till-Bottraud I (2000). Genetic diversity in an endangered alpine plant, Eryngium alpinum L. 
(Apiaceae), inferred from amplified fragment length polymorphism markers. Mol Ecol 9: 162-1637.

Gaudeul M, Naciri-Graven Y, Gauthier P, Pompanon F (2002). Isolation and characterization of microsatellites in a perennial Apiaceae, Eryngium alpinum L. Mol Ecol Notes 2: 107-109.

Gerber S, Mariette S, Streiff R, Bodénès R, Kremer A (2000). Comparison of microsatellites and AFLP markers for parentage analysis. Mol Ecol 9: 1037-1048.

Goudet J (1995). FSTAT vers. 1.2. A computer program to calculate F-statistics. J Hered 86: 485-486.

Haig SM, Gratto-Trevor CL, Mullins TD, Colwell MA (1997). Population identification of western hemisphere shorebirds throughout the annual cycle. Mol Ecol 6: 413-427.

Hedges SB (2000). Human evolution - a start for population genomics. Nature 408: 652-653.

Hedrick PW (1999). Perspective: highly variable genetic loci and their interpretation in evolution and conservation. Evolution 53: 313-318.

Huang BX, Peakall R, Hanna PJ (2000). Analysis of genetic structure of blacklip abalone (Haliotis rubra) populations using RAPD, minisatellite and microsatellite markers. Mar Biol 136: 207-216.

Huff DR, Peakall R, Smouse PE (1993). RAPD variation within and among natural populations of outcrossing buffalograss [Buchloë dactyloides (Nutt.) Engelm]. Theor Appl Genet 86: 927-934.

Hurme P, Savolainen O (1999). Comparison of homology and linkage of random amplified polymorphic DNA (RAPD) markers between individuals trees of Scots pine (Pinus sylvestris L.). Mol Ecol 8: 15-22.

Isabel N, Beaulieu J, Thériault P, Bousquet J (1999). Direct evidence for biased gene diversity estimated from dominant random amplified polymorphic DNA (RAPD) fingerprints. Mol Ecol 8: 477-483.

Jarne P, Lagoda PJL (1996). Microsatellites, from molecules to populations and back. Trends Ecol Evol 11: 424-429.

Jorde PE, Palm S, Ryman N (1999). Estimating genetic drift and effective population size from temporal shifts in dominant gene marker frequencies. Mol Ecol 8: 1171-1178.

Krauss SL (2000). Accurate gene diversity estimated from amplified fragment length polymorphism (AFLP) markers. Mol Ecol 9: 1241-1245.

Krutovskii KV, Erofeeva SY, Aagaard JE, Strauss SH (1999). Simulation of effects of dominance on estimates of population genetic diversity and differentiation. J Hered 90: 499-502.

Lagercranz U, Ellegren H, Andersson L (1993). The abundance of various polymorphic microsatellite motifs differs between plants and vertebrates. Nucleic Acids Res 21: 1111-1115.

Lehmann EL, D'Abrera HJM (1998) In: Nonparametrics: Statistical Methods Based on Ranks. revised edn. Prentice-Hall: Englewood Cliffs, NJ, USA.

Lougheed SC, Gibbs HL, Prior KA, Weatherhead PJ (2000). A comparison of RAPD versus microsatellite DNA markers in population studies of the massasauga rattlesnake. J Hered 91: 458-463.

Lynch M, Milligan BG (1994). Analysis of population genetic structure with RAPD markers. Mol Ecol 3: 91-99.

Maguire TL, Peakall R, Saenger P (2002). Comparative analysis of genetic diversity in the mangrove species Avicennia marina (Forsk.) Vierh. (Avicenniaceae) detected by AFLPs and SSRs. Theor Appl Genet 10: 3-39.

Manel S, Berthier P, Luikart G (2002). Detecting wildlife poaching: identifying the origin of individuals using Bayesian assignment tests and multi-locus genotypes. Conserv Biol 16: 650-659.

Mariette S (2001). Mesure de la diversité génétique intra et interpopulations: échantillonnage intra-génomique et choix des marqueurs. Applications expérimentales sur Quercus petraea, Quercus robur et Pinus pinaster. PhD Thesis. Ecole Nationale du Génie Rural, des Eaux et Forêts: Centre de Paris, France.
Mariette S, Chagné D, Lézier C, Pastuszka P, Raffin A, Plomion $C$ et al. (2001). Genetic diversity within and among Pinus pinaster populations: comparison between AFLP and microsatellite markers. Heredity 86: 469-479.

Mariette S, Cottrell J, Csaikl UM, Goikoechea P, König A, Lowe AJ et al (2002a). Comparison of levels of diversity detected with AFLP and micrsatellite markers within and among mixed Q. petraea (Matt.) Liebl. and Q. robur L. stands. Silvae Genet 5: 72-79.

Mariette S, Le Corre V, Austerlitz F, Kremer A (2002b). Sampling within the genome for measuring within-population diversity: trade-offs between markers. Mol Ecol 11: 1145-1156.

Miller MP (1997). Tools for Population Genetic Analysis (TFPGA), Version 1.3.. Department of Biological Sciences,Northern Arizona University: Arizona, USA.

Milligan BG, McMurray CK (1993). Dominant vs codominant genetic markers in the estimation of male mating success. Mol Ecol 2: 275-283.

Nei M (1987). Molecular Evolutionary Genetics. Columbia University Press: New York, NY.

Paetkau D, Calvert W, Stirling I, Strobek C (1995). Microsatellite analysis of population structure in Canadian polar bears. $\mathrm{Mol}$ Ecol 4: 347-354.

Peakall R, Gilmore S, Keys W, Morgante M, Rafalski A (1998). Cross-species amplification of soybean (Glycine max) simple sequence repeats (SSRs) within the genus and other legume genera: implications for the transferability of SSRs in plants. Mol Biol Evol 15: 1275-1287.

Peakall R, Smouse PE (2001). GenAlEx V5: Genetic Analysis in Excel. Population Genetic Software for Teaching and Research. Australian National University: Canberra, Australia http:/ / www.anu.edu.au/BoZo/GenAlEx/.

Peakall R, Smouse PE, Huff DR (1995). Evolutionary implications of allozyme and RAPD variation in diploid populations of buffalograss Buchloë dactyloides (Nutt. Engelm.). Mol Ecol 4: 135-147.

Pejic I, Ajmone-Marsan P, Morgante M, Kozumplick V, Catiglioni P, Taramino G et al (1998). Comparative analysis of genetic similarity among maize inbred lines detected by RFLPs, RAPDs, SSRs, and AFLPs. Theor Appl Genet 97: 1248-1255.

Powell W, Morgante M, Andre C, Hanafey M, Vogel J, Tingey S et al (1996). The comparison of RFLP, RAPD, AFLP and SSR (microsatellite) markers for germplasm analysis. Mol Breed 2: 225-238.

Raymond M, Rousset F (1995). An exact test for population differentiation. Evolution 49: 1280-1283.

Rieseberg LH (1996). Homology among RAPD fragments in interspecific comparisons. Mol Ecol 5: 99-105.

Roa AC, Chavarriaga-Aguirre P, Duque MC, Maya MM Bonierbale MW, Iglesias C et al (2000). Cross-species amplification of cassava (Manihot Esculenta) (Euphorbiaceae) microsatellites: allelic polymorphism and degree of relationship. Am J Bot 87: 1647-1655.

Ross KG, Dewayne Shoemaker D, Krieger MJB, DeHeer CJ, Keller L (1999). Assessing genetic structure with multiple classes of molecular markers: a case study involving the introduced fire ant Solenopsis invicta. Mol Biol Evol 16: 525-543.

Russell JR, Fuller JD, Macauley M, Hatz BG, Jahoor A, Powell W, Waugh R (1997). Direct comparisons of levels of genetic variation among barley accessions detected by RFLPs, AFLPs, SSRs, and RAPDs. Theor Appl Genet 95: $714-722$.

SAS Institute Inc. (1990). SAS User's Guide. SAS Institute Inc.: Cary, NC, USA.

Streiff R, Labbe T, Bacilieri R, Steinkellner H, Glössl J, Kremer A (1998). Within-population genetic structure in Qercus robur L. and Quercus petraea (Matt.) Liebl. assessed with isozymes and microsatellites. Mol Ecol 7: 317-328. 
Sun GL, Diaz O, Salomon B, Von Bothmer R (1998). Microsatellite variation and its comparison with allozyme and RAPD variation in Elymus fibrosus (Schrenk) Tzvel. (Poaceae). Hereditas 129: 275-282.

Teulat B, Aldam C, Trehin R, Lebrun P, Barker JHA, Arnold GM et al (2000). An analysis of genetic diversity in coconut (Cocos nucifera) populations from across the geographic range using sequence-tagged microsatellites (SSRs) and AFLPs. Theor Appl Genet 100: 764-771.

Thomas BR, MacDonald SE, Hicks M, Adams DL, Hodgetts RB (1999). Effects of reforestation methods on genetic diversity of lodgepole pine: an assessment using microsatellite and randomly amplified polymorphic DNA. Theor Appl Genet 98: 793-801.

Thormann CE, Ferreira ME, Camargo LEA, Tivang JG, Osborn TC (1994). Comparison of RFLP and RAPD markers in estimating genetic relationships within and among cruciferous species. Theor Appl Genet 88: 973-980.
Till-Bottraud I, Gaudeul M (2002). Intraspecific genetic diversity in alpine plants. In: Körner C, Spehn E (eds) Mountain Biodiversity: A Global Assessment. Parthenon Publishing: London, UK.

Ueno S, Tomaru N, Yoshimaru H, Manabe T, Yamamoto S (2000). Genetic structure of Camellia japonica in an old-growth evergreen forest, Tsushima, Japan. Mol Ecol 9: 647-656.

Vos P, Hogers R, Bleeker M, Reijans M, Van de Lee T, Hornes M et al (1995). AFLP: a new technique for DNA fingerprinting. Nucleic Acids Res 23: 4407-4414.

Weir BS, Cockerham CC (1984). Estimating F-statistics for the analysis of population structure. Evolution 38: 1358-1370.

Wyse Jackson PS, Akeroyd JR (1994). Guidelines to be Followed in the Design of Plant Conservation or Recovery Plans. Council of Europe: Strasbourg, France.

Zhivotovsky LA (1999). Estimating population structure in diploids with multilocus dominant DNA markers. Mol Ecol 8: 907-913. 